\title{
EPR in Kagome Staircase Compound $\mathrm{Mg}_{2.997} \mathrm{Co}_{0.003} \mathrm{~V}_{2} \mathrm{O}_{8}$
}

\author{
P. Aleshrevych*, J. Fink-Finowicki and H. Szymczak \\ Institute of Physics, Polish Academy of Sciences \\ al. Lotników 32/46, 02-668 Warsaw, Poland
}

\begin{abstract}
The paramagnetic resonance of $\mathrm{Co}^{2+}$ ions in $\mathrm{Mg}_{2.997} \mathrm{Co}_{0.003} \mathrm{~V}_{2} \mathrm{O}_{8}$ single crystals are reported. The EPR spectrum shows two groups of resonance lines associated with two crystallographically nonequivalent Co ions positions that are known in the kagome staircase system as "cross-tie" and "spine" sites. No preferential occupation of $\mathrm{Co}^{2+}$ ions in the kagome lattice was observed. The ground state was described by spin-Hamiltonian with an effective electronic spin $S=1 / 2$ and nuclear spin $I=7 / 2$. The local symmetry of oxygen octahedron surrounding Co ions, main values of the $g$-factors, and hyperfine structure were determined for both cobalt positions.
\end{abstract}

PACS numbers: 76.30.Da, 76.30.Fc

\section{Introduction}

Magnetic materials with geometrical frustration have recently attracted much interest, both theoretically and experimentally [1]. Of particular interest has been the magnetism on the two-dimensional (2D) kagome lattice because these systems are highly frustrated due to specific geometry. This interest is stimulated by the attempts to find new fundamental effects such as the magnetization plateaus or jumps $[2,3]$. The geometrically frustrated magnets exhibit complex ground states $[4,5]$.

The purpose of this paper is to present the EPR study in $\mathrm{Mg}_{2.997} \mathrm{Co}_{0.003} \mathrm{~V}_{2} \mathrm{O}_{8}$ in which the magnesium sublattice forms the $2 \mathrm{D}$ buckled kagome staircase structure. The investigated single crystals are orthorhombic ( $\mathrm{Cmca}$ space group) with lattice parameters: $a=6.0495(8) \AA, b=11.426(2) \AA, c=8.303(1) \AA[6]$. This compound has the same crystal structure and very similar lattice parameters to the kagome staircase compound $\mathrm{Co}_{3} \mathrm{~V}_{2} \mathrm{O}_{8}$. The fragment of the crystal structure of $\mathrm{Mg}_{2.997} \mathrm{Co}_{0.003} \mathrm{~V}_{2} \mathrm{O}_{8}$ is shown in Fig. 1 . There are two crystallographically nonequivalent positions in $\mathrm{Mg}$ sublattices that are known in the kagome staircase

*corresponding author; e-mail: pavloa@ifpan.edu.pl 
system as "spine" and "cross-tie" sites, which are marked in Fig. 1 as P1 and P2, respectively. The P1 sites form chains along the $a$ axis, which are linked by the P2 sites in the $c$ direction to form the buckled kagome staircase, that are stacked along the $b$ direction. The $\mathrm{P} 1$ and $\mathrm{P} 2$ occupy the centers of the oxygen octahedra. The crystallographic data show that oxygen octahedra around "cross-tie" P2 sites are squeezed along $c$ axis and have an axial symmetry (the distances P2-O4 and $\mathrm{P} 2-\mathrm{O} 5$ are equal to 2.03 and $2.13 \mathrm{~nm}$, respectively). The octahedra around "spine" P1 sites are more distorted (the distances P1-O1, P1-O2, and P1-O3 are equal to $2.11,2.02$, and $2.13 \mathrm{~nm}$, respectively) and also slightly canted from site to site along the $a$ axis. The small amount of cobalt ions is assumed to be in divalent paramagnetic state and replace magnesium ions in the crystal. The $\mathrm{Mg}$ and $\mathrm{V}$ ions are nonmagnetic.

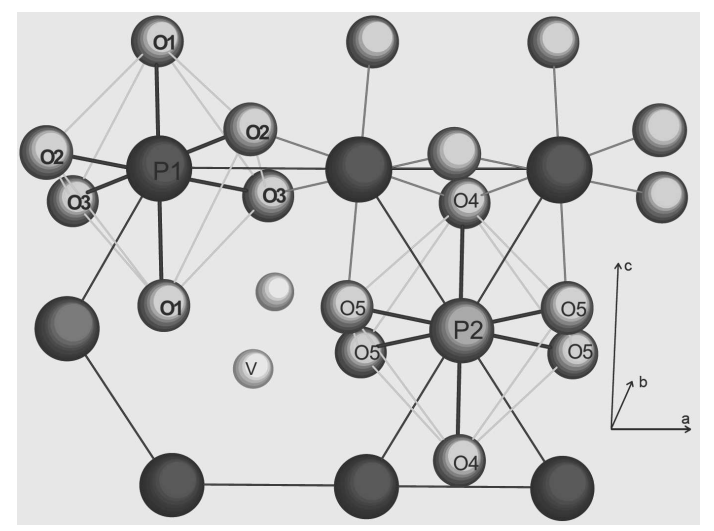

Fig. 1. The crystal structure of the $\mathrm{Mg}_{2.997} \mathrm{Co}_{0.003} \mathrm{~V}_{2} \mathrm{O}_{8}$.

$\mathrm{Co}^{2+}$ in the octahedral field is of particular interest since its ground state is an orbital triplet and the ion has a considerable orbital momentum in cubic crystal field. Therefore, this ion is extremely sensitive to low symmetry fields. Usually a noncubic crystal containing $\mathrm{Co}^{2+}$ shows a large anisotropy of its $g$-factors [7]. The octahedral crystal field acting on $\mathrm{Co}^{2+}$ ion splits the sevenfold degenerate ${ }^{4} F$ state into an orbital triplet ${ }^{4} T_{1}$, followed by another orbital triplet ${ }^{4} T_{2}$ and an orbital singlet ${ }^{4} A_{2}$ [7]. The first excited state of the free $\mathrm{Co}^{2+}$ ion has the ${ }^{4} T_{1}\left({ }^{4} P\right)$ term. The spin-orbit interaction and low symmetry field split the twelvefold degenerate ${ }^{4} T_{1}$ level into a number of the Kramers doublets. The paramagnetic resonance spectra are expected to originate from transitions within the lowest Kramers doublet that is separated by hundreds of wave numbers from the other doublets.

\section{Experimental data and analysis}

The single crystals of $\mathrm{Mg}_{2.997} \mathrm{Co}_{0.003} \mathrm{~V}_{2} \mathrm{O}_{8}$ were grown from polycrystalline feed rods by floating zone technique. The samples were the disks of $3 \mathrm{~mm}$ diameter 
and thickness of $0.5 \mathrm{~mm}$ cut from the single crystalline rod and oriented by X-rays. The EPR measurements were carried out using Bruker EMX spectrometer working on fixed frequency $(9.25 \mathrm{GHz})$ with an Oxford-made helium-flow cryostat working in the temperature range from $3.8 \mathrm{~K}$ to $300 \mathrm{~K}$. The magnetic field modulation at $100 \mathrm{kHz}$ and phase sensitive detection were used to record the derivative of the absorbed microwave power. The samples were glued to a rotating sample holder for the measurements of the angular variation of the EPR spectra.

The EPR spectra were observed at temperatures below $40 \mathrm{~K}$. No EPR lines that could be attributed to $\mathrm{Co}^{2+}$ pairs were observed. The spectrum consists of two groups of eight hyperfine structure components due to ${ }^{59} \mathrm{Co}$ nuclear spin $I=7 / 2$. Figure 2 shows typical EPR spectra of $\mathrm{Mg}_{2.997} \mathrm{Co}_{0.003} \mathrm{~V}_{2} \mathrm{O}_{8}$ for the magnetic field applied parallel to the $c$ axis. The two nonequivalent positions produce the two groups of lines which are interpreted as originating from the transitions between the lowest Kramers doublet $\left(M_{\mathrm{S}}= \pm 1 / 2\right)$ levels. The resonance signal from both groups is very anisotropic. The values of the $g$-factors are practically independent of temperature. It has been shown, based on the analysis of the intensity of EPR lines, that $\mathrm{Co}^{2+}$ ions are distributed randomly. No EPR lines attributed to $\mathrm{Co}^{2+}$ pairs were observed.

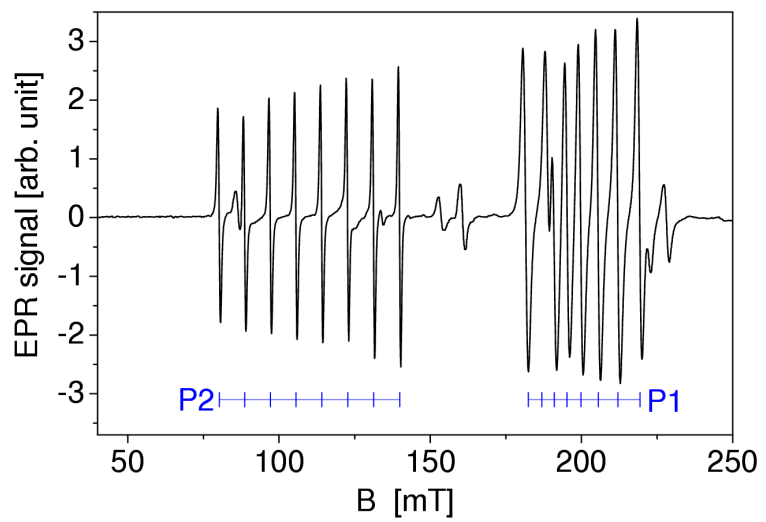

Fig. 2. The EPR spectrum of $\mathrm{Mg}_{2.997} \mathrm{Co}_{0.003} \mathrm{~V}_{2} \mathrm{O}_{8}$ for the magnetic field applied parallel to the $c$ axis at $T=4.2 \mathrm{~K}$.

To find out the main values of $g$ - and $A$-tensors, the angular variations of the EPR spectrum were measured in mutually perpendicular orientations in the planes $a c$ and $b c$ at $4.2 \mathrm{~K}$. The angular dependence of the EPR spectrum in the plane $b c$ is shown in Fig. 3 .

The position of experimental resonance lines can be described by a spin-Hamiltonian of rhombic symmetry with an effective spin $S=1 / 2$ and nuclear spin $I=7 / 2$,

$$
\hat{H}=\mu_{\mathrm{B}}(\boldsymbol{B} g \hat{S})+(\hat{S} A \hat{I})
$$

where $\mu_{\mathrm{B}}$ is the Bohr magneton, $\boldsymbol{B}$ - the magnetic induction vector, $\hat{S}$ - the 


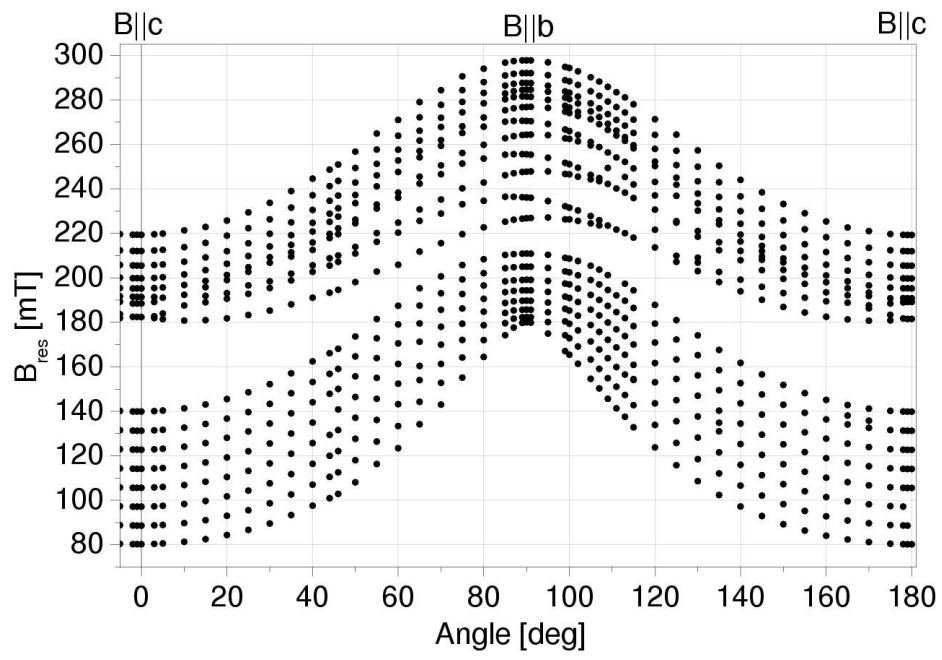

Fig. 3. The angular dependence of the EPR spectrum in the plane $b c$ at $T=4.2 \mathrm{~K}$.

spin operator, $A$ - tensor of the hyperfine interactions, and $\hat{I}$ - the nuclear spin operator. The main values of the $g$ - and $A$-tensors for both positions, determined from the experiment, are listed in Table I.

TABLE I

The main values of the $g$ - and $A$-tensors.

\begin{tabular}{c|c|c|c|c|c|c}
\hline \hline $\begin{array}{c}\text { Posi- } \\
\text { tion }\end{array}$ & $g_{a}$ & $g_{b}$ & $g_{c}$ & $\begin{array}{c}A_{a} \\
{\left[\times 10^{-4} \mathrm{~cm}^{-1}\right]}\end{array}$ & $\begin{array}{c}A_{b} \\
{\left[\times 10^{-4} \mathrm{~cm}^{-1}\right]}\end{array}$ & $\begin{array}{c}A_{c} \\
{\left[\times 10^{-4} \mathrm{~cm}^{-1}\right]}\end{array}$ \\
\hline P1 & $7.038(1)$ & $2.42(1)$ & $3.36(1)$ & $373.6(3)$ & $72.7(7)$ & $79.2(4)$ \\
P2 & $3.247(4)$ & $3.47(1)$ & $6.095(2)$ & $34.8(2)$ & $72.2(4)$ & $242.6(1)$
\end{tabular}

The theory of the paramagnetic resonance of $\mathrm{Co}^{2+}$ in the tetragonal crystal was developed by Abragam and Pryce [8] and extended by Tinkham to the case of the crystal with rhombic symmetry [9]. The components of the $g$-tensor can be written with the accuracy of the first order as follows:

$$
\begin{aligned}
g_{x} & =\left(\frac{10}{3}-\frac{8}{3} \alpha\right)+k(1-2 \alpha), \\
g_{y} & =\left(\frac{10}{3}+\frac{4}{3} \alpha+\frac{4}{3} r\right)+k(1+\alpha+r), \\
g_{z} & =\left(\frac{10}{3}+\frac{4}{3} \alpha-\frac{4}{3} r\right)+k(1+\alpha-r),
\end{aligned}
$$

where $\alpha$ denotes the axial distortion, $r$ is the rhombic distortions, $k$ is the orbital reduction factor corresponding to the reduction of the orbital angular momentum by charge transfer from the paramagnetic ion to the surrounding oxygen ions. 
TABLE II

The values of distortions.

\begin{tabular}{c|c|c|c}
\hline \hline Position & $\alpha$ & $r$ & $k$ \\
\hline P1 & -0.608 & -0.208 & 0.941 \\
P2 & -0.402 & -0.048 & 0.936
\end{tabular}

The values of parameters $\alpha, r, k$ are determined for both positions and listed in Table II.

The value of the factor $k$, smaller than one, shows that in both positions the orbital momentum is partially quenched. However, a big anisotropy of $g$-factor values indicates a still significant contribution of the orbital momentum, also pointing fact that the ground state is an orbital triplet. In spite of the fact that the ionic radius of $\mathrm{Co}^{2+}$ is slightly bigger than the ionic radius of $\mathrm{Mg}^{2+}(0.72 \AA$ and $0.66 \AA$, respectively), the Co replaces $\mathrm{Mg}$ with a very little additional overlap of wave functions, making weak covalent bonding with surrounding oxygen atoms.

The values of parameters ascribing axial and rhombic distortion, together with the crystallographical data, may be used to assign properly the groups of resonance lines to two crystallographic positions. The rhombic distortion is much smaller than axial distortion for "cross-tie" sites. That is why a group of resonance lines with $g$-factor showing nearly axial symmetry $\left(g_{a} \approx g_{b}\right)$ is assumed to belong to cobalt ions in "cross-tie" positions. In the other group of resonance lines all three main values of $g$-factor are different and the rhombic distortion is comparable with the axial distortion. This is expected from crystallographic data for "spine" sites. All three main values of hyperfine interaction tensor are different for both positions.

\section{Conclusion}

The paramagnetic resonance results indicate that the orbital contribution to the magnetic momentum of Co ions is reduced. The local symmetry of oxygen octahedron surrounding Co ions in "cross-tie" and "spine" positions is found to be rhombic. The main values of the $g$-factors and hyperfine structure are determined for both cobalt positions.

\section{Acknowledgments}

The work was partly supported by the State Committee for Scientific Research under the project 1 P03B 03827.

\section{References}

[1] A.P. Ramirez, in: Handbook of Magnetic Materials, Ed. K.J.H. Busch, Vol. 13, Elsevier Science, Amsterdam 2001, p. 423.

[2] H. Kageyama, K. Yoshimura, R. Stern, N.V. Mushnikov, K. Onizuka, M. Kato, K. Kosuge, C.P. Slichter, T. Goto, Y. Ueda, Phys. Rev. Lett. 82, 3168 (1999). 
[3] R. Szymczak, M. Baran, R. Diduszko, J. Fink-Finowicki, M. Gutowska, A. Szewczyk, H. Szymczak, Phys. Rev. B 73, 1 (2006).

[4] B. Canals, C. Lacroix, Phys. Rev. Lett. 80, 2933 (1998).

[5] A.P. Ramirez, A. Hayashi, R.J. Cava, R. Siddhartan, B.S. Shastry, Nature 399, 333 (1999).

[6] J.D. Pless, N. Erdman, D. Ko, L.D. Marks, P.C. Stair, K.R. Poeppelmeier, Crystal Growth \& Design 3, 615 (2003).

[7] A. Abragam, B. Bleaney, Electron Paramagnetic Resonance of Transition Ions, Clarendon Press, Oxford 1970.

[8] A. Abragam, M.H.L. Pryce, Proc. R. Soc. Lond. A 206, 173 (1951).

[9] M. Tinkham, Proc. R. Soc. Lond. A 236, 549 (1956). 